\title{
Giant left atrial appendage
}

\author{
W. Tietge, L.C. Otterspoor, R. Uylings, G.Tj. Sieswerda, M.J.M. Cramer, P. Loh
}

A 42-year-old man was evaluated at our hospital because of paroxysmal atrial fibrillation. Physical examination and ECG during sinus rhythm were unremarkable (no signs of left atrial enlargement).

Further evaluation with transoesophageal echocardiography showed a giant left atrial appendage (figures 1 and 2). No left atrial or left atrial appendage (LAA) thrombi were seen, there was no spontaneous echo contrast and LAA flow

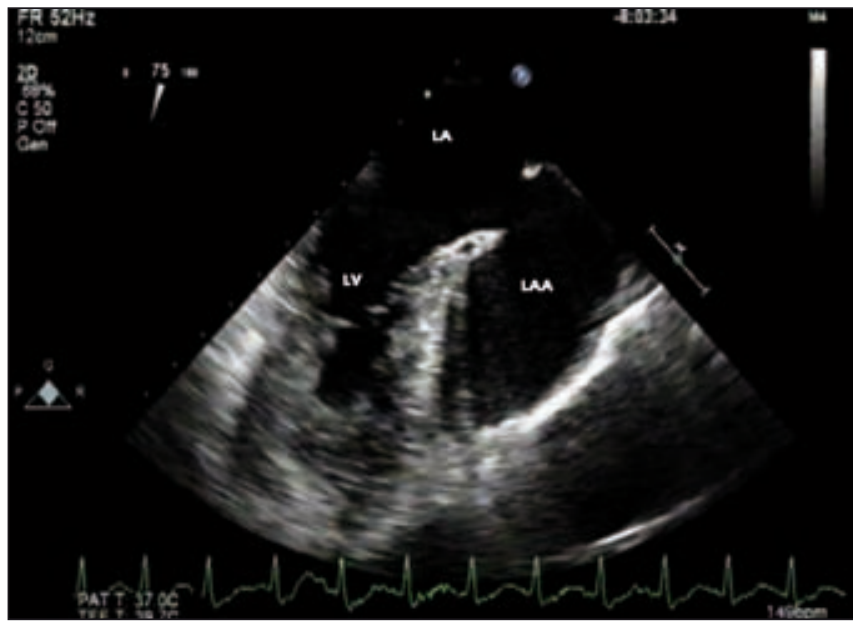

Figure 1. Transoesophageal echocardiography during systole. $L V=$ left ventricle, $L A=$ left atrium, $L A A=$ left atrial appendage, $M V=$ mitral valve.

\section{W. Tietge}

L.C. Otterspoor

R. Uylings

G.Tj. Sieswerda

M.J.M. Cramer

P. Loh

Department of Cardiology, University Medical Center Utrecht, Utrecht, the Netherlands

Correspondence to: W. Tietge

Department of Cardiology, University Medical Center Utrecht, PO Box 85500, 3508 GA Utrecht, the Netherlands

E-mail: wouter@tietge.nl velocities were normal. No other structural or functional abnormalities were observed. A giant left atrial appendage is not frequently described according to the current literature and is regarded as a congenital heart defect. It has been associated with supraventricular arrhythmias, thromboembolism, cardiac dysfunction, mitral regurgitation and/or compression of the left anterior descending artery. ${ }^{1-3}$

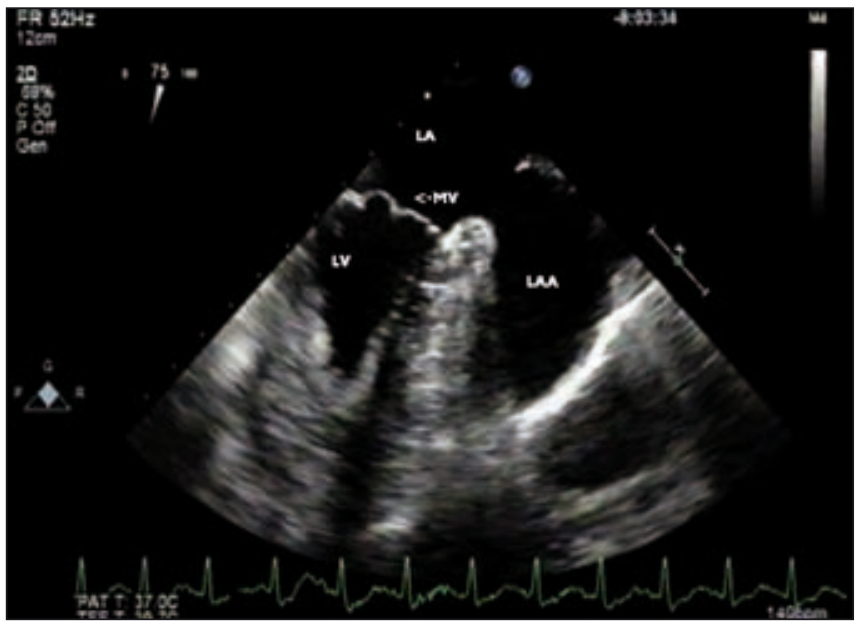

Figure 2. Transoesophageal echocardiography during diastole. $L V=$ left ventricle, $L A=$ left atrium, LAA=left atrial appendage.

\section{References}

l Gold JP, Afifi HY, Ko W, Horner N, Hahn R. Congenital giant aneurysms of the left atrial appendage: diagnosis and management. J Card Surg. 1996;11:147-50.

2 Acartürk E, Kanadasi M, Yerdelen VD, Akpinar O, Ozeren A, Saygili OB. Left atrial appendage aneurysm presenting with recurrent embolic strokes. Int J Cardiovasc Imaging. 2003;19:495-7.

3 Rikikitake K, Minato N, Ohnishi H, et al. Mitral valve replacement through a giant left atrial appendage. J. Cardiovasc Surg. 1999;40:127-9 